\title{
Multi-Compartment Centrifugal Electrospinning Based Composite Fibers
}

\section{Li Wang ${ }^{a, b}$, Zeeshan Ahmad ${ }^{c}$, Jie Huangd, Jing-Song Lia ${ }^{a}$, Ming-Wei Chang ${ }^{a, b, c *}$}

${ }^{a}$ Key Laboratory for Biomedical Engineering of Education Ministry of China, Hangzhou, 310027, P. R. China.

${ }^{\mathrm{b}}$ Zhejiang Provincial Key Laboratory of Cardio-Cerebral Vascular Detection Technology and Medicinal Effectiveness Appraisal, College of Biomedical Engineering \& Instrument Science, Zhejiang University, Hangzhou, 310027, P. R. China.

c College of Biomedical Engineering \& Instrument Science, Zhejiang University, Hangzhou, 310027, P. R. China.

${ }^{\mathrm{d}}$ Leicester School of Pharmacy, De Montfort University, The Gateway, Leicester, LE1 9BH, UK.

e Department of Mechanical Engineering, University College London, London WC1E 7JE, UK. 
CMCCE: combinatorial multi-compartment centrifugal electrospinning system

CES: centrifugal electrospinning system

CO-ES: co-axial electrospinning

SEM: scanning electron microscopy

FTIR: fourier transform infrared spectroscopy

EE: encapsulation efficiency

PS: polystyrene

TPU: thermoplastic polyurethane

DCM: dichloromethane

TE-HCl: hydrochloride
ES: electrospinning

OM: optical microscopy

FM: fluorescence microscopy

FFT: fast Fourier transform

PVP: polyvinyl pyrrolidone

PCL: polycaprolactone

DMAc: N,N-dimethylformamide

EtOH: ethanol

PBS: phosphate buffer saline 


\begin{abstract}
Multi-faceted technological advances in fiber science have proven to be invaluable in several emerging biomaterial and biomedical engineering applications. In the last decade, notable fiber engineering advances have been demonstrated ranging from co-axial flows (for micron and nano-scaled layering), non-concentric flows (for Janus composites) and even 3D printing (for controlled alignment). The ES process is however limited, both for commercial impact (low production rates) and also in its facile capability to deliver reliable mimicry of numerous biological tissues which comprise blended and aligned fibers (e.g. tendons and ligaments). In the technological advance demonstrated here, a combinatorial multi-compartment centrifugal electrospinning (CMCCE) system is developed and demonstrated. A proof-of-concept enabling multiple formulation solution hosting (including combinatorial grading) in a single centrifugal electrospinning system (CES) comprising one spinneret is shown. Using this process, controlled blending and tuning of resulting fibrous membrane properties (contact angle and active release behavior) via aligned and phased fiber mat composition is demonstrated. In addition, the CMCCE process is capable of replicating production rates of recently developed centrifugal electrospinning systems $(\sim 120 \mathrm{~g} / \mathrm{h})$, while potentially permitting better mimicry of naturally occurring fibrous tissue blends. It is envisaged the advance in technology will be ideally suited to engineer synthetic fibrous biomaterials with greater host surface replication and will fulfil production rate requirements for the industrial sector.
\end{abstract}

Key words: centrifugal electrospinning; multi-compartment; composition; combinatorial; fiber. 


\section{Introduction}

The last two decades have experienced a multi-faceted re-emergence in fiber science $[1,2]$. Much of this progress is directly attributed to advances in other distinctly separate fields (e.g. biomedical engineering, environment, energy and pharmaceutical sciences) [3-6]. Several intrinsic features of fibrous materials and resulting bulk systems (surface, macro-scale morphology and geometrical bundling or patterning) have propelled them as ideal platforms to address numerous global challenges [7-9]. This, in turn, has driven the development of fiber engineering or yielding methods; providing opportunities to scale down in fiber size, mass produce at the ambient environment, in lieu alignment during forming and to host a wide range of functional, sensitive or active materials within their filamentous structure $[10,11]$. Co-axial electrospinning (CO-ES), centrifugal electrospinning (CES), fiber printing (2D and 3D) rotary jet-spinning are notable advances within this remit [12-14]. These developments have enabled multi-phase fiber composites and compartmentalization [15], ordered filamentous structures [16], Janus fiber systems [17] and high production rates [18]. It is noteworthy, technological advance is often driven by the underlying need or property requirement of an end application; and electrospinning (ES), and its various sister processes (i.e. electrospraying), have been explored in great depth for biomaterial and biomedical material purposes, primarily due to their facile operation, process control, ambient temperature operation and fiber size scalability $[19,20]$. There is a clear expectation that these (and related technologies) will converge, enhance and diversify, as needed, over time [21].

Engineered fibrous materials enable numerous benefits for various bio-related explorations and investigations. For example, fibrous structures exist naturally within the body as both soft and hard tissues (e.g. muscle [22, 23] comprise red type I (hard) and white type II (soft) fibers. Red fibers tend to have more mitochondria and greater local capillary density, which are more suited for endurance and slow to fatigue. White fibers 
have high glycolytic enzymes and fast twitch properties. The two components are networked in muscle, which causes muscle contraction. Using this as an example, concept development based on biomimetics is possible (e.g. mimicking the extra cellular matrix). While ES has been the preferred fabrication method to show proof of concepts relating to fibrous biomaterials [24], there are several drawbacks which limit commercial impact and also real in vivo replication $[25,26]$. For example, bionic tissue is a blend of multi-aligned fibers (e.g. cardiovascular [27], tendon [28] and ligament [29]), and the vast majority of ES explorations have focused on mono-fiber matrices with little to no orientation. Furthermore, bionic tissue inspired architectures also give rise to intricate surface patterning aspects, further matching real tissue functionality [30]. Together with naturally occurring fiber and industrial blending and alignment, these constitute towards vital fiber components [31]. In this regard, synthetic design, construction and engineering of aligned fiber blends is crucial to further advance potential biomaterial systems [32, 33].

Where ES has been used to blend fibers the yield has been appreciably low [34]. Recent advances have, however, led to the development of CES. Here a centrifugal drawing force is utilized alongside the conventional electrical force to improve both yield and alignment. Both forces stretch and elongate the liquid polymer jet in CES to form fibers. CES has a broader range of 'spinnable' materials and is applicable for both conductive and nonconductive polymeric solutions [35], and process parameters include rotation speed, voltage and orifice size. Fiber production rates using CES (using a needle-less system) have reached $120 \mathrm{~g} / \mathrm{hr}$ [36]. Multi-fiber blending via CES is scarce and conventionally a single medium hosting compartment is used.

To improve fibrous biomaterial mimicry and aligned-blend fiber production rate in tandem the present work demonstrates the development of an advanced CES system deploying for the first time multiple media hosting compartments (exemplified using $n=4)$. A combinatorial approach in material selection is shown which provides a route to 
blend and engineer aligned mat compositions from multiple fibers. To elucidate material applicability further, polymers polyvinyl pyrrolidone (PVP), polystyrene (PS), polycaprolactone (PCL) and thermoplastic polyurethane (TPU) were used to synthesize multi-material stacked aligned fibers. Finally, the impact of combinatorial material selection is demonstrated through tuning active release rate (from blended membranes) and contact angle behavior, both of which are valuable properties of biomaterials interacting at a physiological interface. Biomaterial contact angle has significant impact on cellular interactions [37] and the materials surface is the first point of interaction with biological (host) surroundings. Thus, wettability is influenced primarily by surface characteristics which in turn impacts bio-interface behavior [38]. In addition, wettability has also been shown to modulate hydrophobicity, which is an important parameter in drug release from the delivery system [39].

\section{Materials and Methods}

2.1. Materials and solution synthesis

Polyvinyl pyrrolidone (PVP, mean $M_{w}=1.3 \times 10^{6} \mathrm{~g} / \mathrm{mol}$ ), Polystyrene (PS, mean $M_{\mathrm{w}}=1.92 \times 10^{5} \mathrm{~g} / \mathrm{mol}$ ), Polycaprolactone (PCL, mean $M_{w}=8 \times 10^{5} \mathrm{~g} / \mathrm{mol}$ ), Rhodamine B (mean $M_{w}=479.01 \mathrm{~g} / \mathrm{mol}$ ) were purchased from Sigma-Aldrich Ltd., St Louis, USA. Thermoplastic polyurethane (TPU) was purchased from Solvay China Ltd., Shanghai, China. Acriflavine (mean $M_{w}=259.73 \mathrm{~g} / \mathrm{mol}$ ) was purchased from Macklin, Shanghai, China. N,N-dimethylformamide (DMAc), dichloromethane (DCM) and ethanol (EtOH) were purchased from Sinopharm Chemical Reagent Co., Ltd, Beijing, China. Tetracycline hydrochloride (TE-HCl) was purchased from Amresco, USA. Phosphate buffer saline (PBS) was supplied by Sinopharm Chemical Reagent Co., Ltd, Shanghai, China. All materials were of the analytical grade and were used as received without further purification. 
PVP solutions (ranging from 0 to $25 \mathrm{wt} . \%$ ) were prepared by dissolving the polymer in ethanol with continuous mechanical stirring (VELP ARE heating magnetic stirrer, Italy) at $25{ }^{\circ} \mathrm{C}$ for $3 \mathrm{~h}$ to ensure complete dissolution. PS solutions (ranging from 0 to $30 \mathrm{wt} . \%$ ) were prepared by dissolving the polymer in DMAc with continuous mechanical stirring at $50{ }^{\circ} \mathrm{C}$ for $6 \mathrm{~h}$ to ensure complete dissolution. The $8 \mathrm{wt} \%$ PCL solution (in DCM) was prepared by magnetic stirring at $50{ }^{\circ} \mathrm{C}$ for $6 \mathrm{~h}$ with dichloromethane. TPU (20 wt. $\%$ ) was dissolved in DMAc through magnetic stirring for $6 \mathrm{~h}$ at $50{ }^{\circ} \mathrm{C}$.

\subsection{System setup}

The complete CMCCE system is shown in Fig. 1(a). It comprises a conductive iron collector (wire diameter $=0.5 \mathrm{~mm}$, collector diameter from central spinneret point $=18 \mathrm{~cm}$ ) and an ES rotating spinneret as shown in Fig. 1(b) which hosts individual solution compartments; with two detached media hosting channels displayed in the graphic. The spinneret is $80 \mathrm{~mm}$ in length and possesses upper diameter of $30 \mathrm{~mm}$. The volume of each compartment is $2 \mathrm{~mL}$ with a diameter of $11 \mathrm{~mm}$ and $41 \mathrm{~mm}$ length. The electrospinning pore is placed on the bottom of the compartment, and the diameter of the pore can adjust from $0.4 \mathrm{~mm}$ to $0.6 \mathrm{~mm}$. The spinneret system is capable of hosting numerous individual polymer solution compartments (or any other ESp material e.g. suspension, slurries and emulsions) as shown in Fig. 1(c). The collector was positioned around the spinning system at the perimeter, and by applying a DC voltage of $12-18 \mathrm{kv}$ (maximum $\sim 30 \mathrm{kV}, 0.1 \mathrm{~mA}$ ) (Glassman high voltage Inc. series FC, USA) and a rotation speed varying from $200 \mathrm{rpm}$ to $400 \mathrm{rpm}$ (maximum $\sim 15000 \mathrm{rpm}$ ). The relationship between resulting fiber properties and parameters were investigated systematically.

\subsection{Characterization}

2.2.1. Viscosity measurements. A Brookfield viscometer (DV2TLV, Brookfield, USA) was used to measure polymer solution viscosity. PVP and PS solutions were placed into 
the sample vial and viscosity measurements were obtained suing a plate spindle system (CPA-41Z, $48 \mathrm{~mm}$ in diameter and $0.4 \mathrm{~mm}$ in gap distance). Viscosities were recorded under steady-state shear stress from 0.003 to $40 \mathrm{~Pa}$ at the ambient temperature $\left(25{ }^{\circ} \mathrm{C}\right)$. 2.2.2. Optical microscopy (OM). An optical microscope (OM) (Pheonix BMC503-ICCF, China) was used to characterize morphology and arrangement of fibers engineered using CMCCE. The optical images were further processed for backlight and alignment analysis. .

2.2.3. Scanning electron microscopy (SEM). Electron micrographs were obtained using a scanning electron microscope (Hitachi, Japan). Prior to SEM analysis, samples were sputter-coated (Sputter coater 108 auto, Cressington Scientific Instruments Ltd., UK) with Pt for $90 \mathrm{~s}$ at a current intensity of $25 \mathrm{~mA}$. All samples were analyzed at an accelerating voltage of $20 \mathrm{kV}$ and $500 \times$ magnification.

2.2.4. Fluorescence microscopy (FM). Fluorescent micrographs were obtained using an inverted microscope (Eclipse Ti-S, Nikon, Tokyo, Japan) equipped with camera (DS-Qi2, Nikon, Tokyo, Japan). All samples were observed at 10× magnification. Polymer solutions were labeled through addition of $1 \mathrm{w} / \mathrm{v} . \%$ of selected fluorescent probe acriflavine (green, excitation in $440 \mathrm{~nm}$ ) or rhodamine-B (red, excitation in $540 \mathrm{~nm}$ ).

2.2.5.Fourier transform infrared spectroscopy (FTIR). FTIR (IR Affinity 1, Shimadzu, Japan) was used to determined sample composition. Prior to analysis, samples were prepared using the $\mathrm{KBr}$ pellet pressing method. Here, $2 \mathrm{mg}$ of engineered fibers were dispersed in $200 \mathrm{mg}$ of $\mathrm{KBr}$ medium through grinding and were then compressed into transparent pellets $(20 \mathrm{MPa})$. Spectra were obtained at a resolution of $4 \mathrm{~cm}^{-1}$ (over the $4000-400 \mathrm{~cm}^{-1}$ region).

\subsection{Drug release behavior.}

Antibiotic release from selected aligned fibers systems was demonstrated using UV spectroscopy. A UV spectrophotometer (UV-2600, Shimadzu, Japan) was used to 
generate a linear calibration curve of standard TE-HCL solutions ranging from 5-100 $\mu \mathrm{g} / \mathrm{mL}$. For TE-HCL release studies, $1 \mathrm{~mL}$ of supernatant was removed for $\mathrm{UV}$ measurements and an equal volume of fresh release medium was added to the test medium. TE-HCL concentration was determined using a wavelength of $356 \mathrm{~nm}$. Selected fibrous samples were immersed in a sealed bottle containing $100 \mathrm{~mL}$ of PBS at a temperature of $37^{\circ} \mathrm{C}$. Sample vials were agitated (200 rpm) using a HZ-8801K thermostatic oscillator (Taicang Science and Education Factory, China).

Eqn. (1) was used to determine the cumulative release of TE-HCL ( $\left.{ }^{\eta}\right)$.

$$
\eta_{\%}=m / M \times 100
$$

Where $m$ is quantity of TE-HCL in the solution at specified time points and $M$ is the maximum quantity of TE-HCL in the solution at infinite time.

For encapsulation efficiency (EE) studies $50 \mathrm{mg}$ of formulated drug loaded fiber was completely dissolved in $20 \mathrm{ml}$ corresponding solvents. Thereafter the TE-HCl concentration was determined using UV spectrophotometry.

Eqn. (2) was used to calculate the encapsulation efficiency (EE).

$$
\mathrm{EE} \%=\mathrm{P}_{\mathrm{t}} / \mathrm{L}_{\mathrm{t}}
$$

Where $\mathrm{P}_{\mathrm{t}}$ is the quantity of TE- $\mathrm{HCl}$ embedded in fibers and $\mathrm{L}_{t}$ is the theoretical quantity of $\mathrm{TE}-\mathrm{HCl}$ (from the feeding solution) incorporated into the polymeric system.

\subsection{Electric field analysis}

Electric field computations were performed using Comsol Multiphysics 5.2 via finite element method. Three-dimensional geometries of the device was developed with computational boundaries using COMSOL Multiphysics [40, 41]. The working distance 
and applied voltage parameters were set to $12 \mathrm{~cm}$ and $18 \mathrm{kv}$, respectively.

\subsection{Image processing.}

ImageJ software (National Institute of Health, USA) was used to analyze micrographs (for mean fiber diameter) enabling statistical distribution measurements. Here, each experiment involved a random sample of 100 fibers. Origin software (OriginLab, USA) exported all data for further analysis while plotting graphs. Error bars were plotted to represent the mean \pm standard deviation. Fast Fourier transform (FFT) was performed using MATLAB (MathWorks, Natick,Massachusetts, USA) to determine fiber alignment based on binary $\mathrm{OM}$ micrographs to confirm the relative degree of fiber alignment via conversion of fiber location image into spacing frequency.

\section{Results and discussions}

\subsection{Centrifugal electrospinning system}

The complete CMCCE system is shown in Fig. 1(a). It comprises a collector (conductive iron wire (wire diameter $=0.5 \mathrm{~mm}$, collector diameter from central spinneret point $=18 \mathrm{~cm}$ ) and an ES rotating spinneret as shown in Fig. 1(b) which hosts individual solution compartments; with two detached media hosting channels displayed in the graphic. The spinneret system is capable of hosting numerous individual polymer solution compartments (or any other ESp material e.g. suspension, slurries and emulsions) as shown in Fig. 1(c), although there is no restriction on compartment volume, number, size or orientation. In fact, the centrifugal element (force) enables utilization of non-ESp materials too and enhances production rates. The electric field (Fig. 1(d)) is the main driving force for electrically conducting (ESp) materials, and ultimately permits 'jet' formation, fiber trajectory and deposition towards the collector frame. Fig. 1(e) illustrates multi-fiber blending (shown using two compartments) to yield a composite fibrous membrane. Blended fibers have potential to overcome limitations (multi-step engineering, 
poor biological mimicry and limited control on charged fiber interaction) often associated with conventional ES methods. A combinatorial approach based on compartment number and deployed material type provides extensive scope for composite material development and functional property fine-tuning. Fig. 1(f) shows a fluorescent micrograph of a fibrous composite engineered using CMCCE, where PS (acriflavine probe, green) and PVP (rhodamine-B probe, red) are spun (both solutions display separate jetting and fiber formation) in tandem hosting specific fluorescing materials. The impact of specific conventional ES process parameters (such as flow rate and ambient pressure) is reduced in $\mathrm{CMCCE}$ which enhances the overall process reliability. Furthermore, the added benefit of alignment, controlled blending and greater production rate are achievable.

In this conceptual study, highly aligned fibers were generated using CMCCE deploying a spinneret rotational speed of $400 \mathrm{rpm}$ and an applied voltage of $18 \mathrm{kV}$. For the initial exploration, two spinneret compartments were used. Fig. 2(a) exhibits aligned PS fibers possessing a mean diameter of $25.5 \pm 9.9 \mu \mathrm{m}$. In this instance PS solution $(22.5$ wt.\%) was spun from both compartments. Under identical (aforementioned) conditions, highly aligned fibers also result when PVP solution (20 wt.\%) replaces PS in both compartments, although there is a reduction in the mean fiber diameter (Fig. 2(b), 12.4 $\pm 4.0 \mu \mathrm{m})$. Membranes comprising both PS and PVP aligned fibers are generated when they are deployed at a 1:1 compartment ratio (Fig. 2(c)). PS fibers exhibit reduced light transmission compared to PVP fibers, which results in dark (PS) and bright (PVP) filaments as observed in optical micrographs. All membranes comprise highly aligned fibers with smooth surface as evidenced in electron micrographs (Fig. 2(d)-(f)). Individual and over-layered fluorescent micrographs are shown in Figs. 2(g)-(i). Figs. 2(g) and (h) show fluorescence from entrapped probe ( 1 w/v.\%) within PS (acriflavine probe, green) and PVP (rhodamine-B probe, red), respectively, indicating the presence and location of each fiber type. Fig. 2(i) is an over-layered fluorescent micrograph confirming the simultaneous engineering of PS and PVP fibers through CMCCE, while at the same 
time clearly indicating active material retention and active carrier potential. Corresponding mean fiber diameter distributions and water contact angles (as in-sets) are shown in Figs. 2(j)-(1). PVP fibers possess smaller diameters giving rise to a clear bi-modal fiber distribution in the blended composite system. Differences in PS (hydrophobic) and PVP (hydrophilic) fibrous membrane hydrophobicity is as one would expect $\left(100.9 \pm 0.7^{\circ}\right.$ and $47.6 \pm 1.0^{\circ}$, respectively), and the impact of a blended system shows a medium contact angle $\left(67.9 \pm 0.6^{\circ}\right)$, which combines the hydrophilic-hydrophobic property of both materials, indicating a method to modulate surface wettability. Furthermore, compartment solution allocation and individual polymer solution concentration are other parameters which can further optimize contact angle behavior which is crucial for cell bio-interface applications such as bionic tissue development [42].

\subsection{Congruence and parametric control}

In this study, two polymeric solutions were used simultaneously as separate compartments within the hosting CES spinneret. The relationship between two simultaneously jetting and (eventually) adjacently forming and aligned fibrous materials was explored. Therefore, establishing a quantifiable model to deduce viable process parameters (e.g. solution viscosity, rotation speed, applied voltage) becomes vital. Fig. 3(a) demonstrates the relationship between solution viscosity and process polymer solution concentration; as importantly maps onto this relationship are key fiber forming zones. As demonstrated in a previous study [36], a critical polymer concentration must be reached for pristine fiber production, denoted as entanglement concentration (T). This is identified as the intersection point between good (pristine fibers exhibiting near uniform morphology) and poor fiber (fibers displaying beaded and other irregular inconsistencies such as varying diameter along a fibers' axial length) production as shown in Fig. 3(a). Therefore, critical polymer loading concentrations for selected solutions were determined 
to be 19 wt.\% for PVP (in EtOH) and 24 wt.\% for PS (in DMAc). The critical concentration can also be approximated using a viscosity plot and by identifying the sharp shift in viscosity as a function of polymer concertation [36]. When the polymer concentration in solution is low, chain entanglement is not sufficient to promote chain overlap, which means fiber formation is not achievable or is poor [43]. When the solution concentration reaches and surpasses $(\mathrm{T})$ value, intermolecular forces enable pristine fiber formation. In the current study, the explored PVP concentration ranged from 16 to 24 wt.\% and from 20 to $27.5 \mathrm{wt} \% \%$ for PS polymer.

Once the minimal polymer concentration was determined process parameters (such as spinneret rotation speed and the applied voltage) were investigated. For this, identical spinneret and compartments were used (materials, geometry, volume and orientation). Fig. 3(b) illustrates the relationship between applied voltage and deployed rotation speed for fiber production. In the first instance, PVP solution (19 wt.\%) was subjected to numerous preliminary experiments to determine minimum working parameters for fiber formation. This boundary was determined experimentally and is shown as the black curve; indicating process viability at $10.0 \mathrm{kV}$ and $100 \mathrm{rpm}$ to $6.0 \mathrm{kV}$ and $400 \mathrm{rpm}$. Numerical parametric adjustments to these values must be above this curve in order to yield aligned PVP fibers. The red curve is the operational boundary (minimal process parameters) for 24 wt.\% PS solution. Here the process viability was between $18.5 \mathrm{kV}$ and $100 \mathrm{rpm}$ to 12 $\mathrm{kV}$ and $400 \mathrm{rpm}$. The shaded zone in Fig. 3(b) appears above both curves and is the optimal blended aligned fiber-engineering region for both simultaneously jetting polymer solutions. The combinatorial parametric relationship also suggests a compensating effect exists. At a comparatively low applied voltage, higher rotation speeds enable fiber formation and vice versa. In addition, orifice diameter can be used to modulate fiber formation and this was explored using two orifice diameters.

The relationship between parametric control and fiber diameter was investigated further. A selection of PS (range 20-27.5 wt.\%) and PVP (16-24 wt.\%) solutions with 
varying concentrations were deployed according to the determined critical viscosity values. An increase in PS solution concentration leads to a reduction in fiber diameter as shown in Fig. 3(c). For PS, deploying a polymer solution concentration of 20, 22.5, 25 and 27.5 wt.\%, yields fibers with mean diameters of 13.0 $\pm 4.9,25.5 \pm 9.9,22.6 \pm 4.0$ and $18.4 \pm 3.8 \mu \mathrm{m}$, respectively. For the last three concentrations, the size reduction could be ascribed to greater polymeric concentrations impacting reduced solvent and solvent evaporation rates resulting in finer fibers [44]. A clear trend, however, is observed in resulting fiber diameter with increasing PVP solution concentration. Mean fiber diameters were $45.5 \pm 14.4,27.8 \pm 5.9,12.4 \pm 4.0,10.4 \pm 3.0$ and $16.0 \pm 4.6 \mu \mathrm{m}$ for $16,18,20$, 22 and 24 wt.\% PVP solution concentrations, respectively. An anomaly for the 24 wt.\% solution is ascribed to deviation in critical viscosity. Fig. 3(d) illustrates the impact of compartment orifice size (media ejecting point during CMCCE) on resulting fiber diameter. PS fiber diameter decreased from $52.9 \pm 11.3$ to $22.6 \pm 4.1 \mu \mathrm{m}$ and a similar trend was observed for PVP polymer (i.e. $46.4 \pm 12.9$ to $12.4 \pm 4.0 \mu \mathrm{m}$ ) when the orifice diameter was reduced from 0.6 to $0.4 \mathrm{~mm}$. A reduction in the compartment orifice leads to greater fiber uniformity and facilities finer fiber formation.

For spinneret rotating speeds, mean fiber diameters of PS were 11.5 $\pm 4.2,20.2 \pm 6.7$ and 22.6 $\pm 4.0 \mu \mathrm{m}$ (rotational speeds of 200, 300 and $400 \mathrm{rpm}$, respectively) as shown in Fig. 3(e). A similar trend is observed for PS fibers. Increasing the rotation speed leads to coarser fiber engineering. A greater rotation speed accelerates liquid ejection from the compartments orifice. This in turn leads to an increased fiber diameter. Fig. 3(f) demonstrates the impact of applied voltage on fiber diameter. Mean PS fiber diameters are $38.5 \pm 6.6,37.0 \pm 13.5,29.7 \pm 7.6,26.0 \pm 8.8,22.6 \pm 4.0 \mu \mathrm{m}$ at an applied voltage of 12 , 13.5, 15, 16.5 and $18 \mathrm{kV}$, respectively. An increase in applied voltage provides an enhanced electrical force, contributing towards fiber elongation, thus decreasing fiber diameter. However, mean fiber diameters for PVP formulations are 9.9 $\pm 4.9,14.7 \pm 3.8$, $7.3 \pm 2.1,11.0 \pm 3.5,12.4 \pm 4.0 \mu \mathrm{m}$ at an applied voltage of $12,13.5,15,16.5$ and $18 \mathrm{kV}$, 
respectively. An increase in mean fiber diameter is observed when the electrical field was increased from 12 to $13.5 \mathrm{kV}$, although a reduction was observed thereafter. This anomaly is explained due to solution viscosity. The selected PVP solution has a viscosity of 7044 $\mathrm{mPa} \cdot \mathrm{s}$, which is higher than the selected PS solution (2039 $\mathrm{mPa} \cdot \mathrm{s})$. The impact of centrifugal force is more pronounced on media with lower viscosity.

\subsection{FTIR spectroscopy}

FTIR was used to confirm PVP and PS fiber blends, focusing in the $4000-400 \mathrm{~cm}^{-1}$ region. Spectra for PVP, PS, TE-HCl, PVP \& TE-HCl, PS \& TE-HCl, PS \& PVP \& TE-HCl are displayed in Fig. 4(a). For PS, bands at $2922 \mathrm{~cm}^{-1}$ are assigned to asymmetric stretching vibrational modes of the $\mathrm{CH}_{2}$ group, and the band at $1600 \mathrm{~cm}^{-1}$ is due to $\mathrm{C}-\mathrm{C}$ stretching vibrational mode for the phenyl group [45]. Characteristic absorption peaks at 1658 and $1288 \mathrm{~cm}^{-1}$ are observed for $\mathrm{PVP}$, which are assigned to $\mathrm{C}=\mathrm{O}$ and $\mathrm{C}-\mathrm{N}$ vibrations, respectively [46]. The spectra for $\mathrm{TE}-\mathrm{HCl}$ shows two characteristic IR regions; at 1460 $\mathrm{cm}^{-1}\left(\mathrm{OH}\right.$ bending) and 1250-1200 $\mathrm{cm}^{-1}$ (C-C stretching and bending, $\mathrm{N}-\mathrm{H}$ bending and C-N stretching). Compared to pristine polymers, spectra for mats prepared using PVP \& TE-HCL, PS \& TE-HCL, PS \& PVP \& TE-HCL exhibit characteristic peaks for the active at 1460 and $1228 \mathrm{~cm}^{-1}$ [30]. No other absorption bands were detected in resulting drug-fibrous samples, confirming no new chemical bond formation or significant intermolecular interactions arising within blended fibers.

\subsection{In vitro drug release study}

Drug release behavior from various aligned fibrous systems was investigated. Three fibrous drug loaded samples (PVP, PS and PS \& PVP) were prepared using CMCCE under optimized working conditions (400 rpm and $18 \mathrm{kV}$ ). Active release behavior was evaluated in vitro $(\mathrm{PBS}, \mathrm{pH}=7.4)$ at ambient conditions $\left(37^{\circ} \mathrm{C}\right)$. In addition, a PS solution concertation of 22.5 wt. \% was selected (compartmental ejecting orifice $=0.6$ 
$\mathrm{mm}$ ). PVP samples were prepared using a $20 \mathrm{wt} \%$ solution (compartmental orifice $=0.4$ $\mathrm{mm})$. The drug encapsulation efficiency of PVP, PS and PS \& PVP was 98.8 $\pm 1.1,96.3$ $\pm 0.9,97.1 \pm 1.5$, respectively. All three samples show high active entrapment (>95\%), indicating the CMCCE process is also economical in terms of efficiency.

Near complete TE-HCL release from PVP fibers $(97.9 \pm 1.9 \%)$ was achieved in 30 minutes, while release from PS matrices only reached $8.9 \pm 1.9 \%$ at the same time interval, which approximately doubles (18.3 $\pm 1.2 \%)$ at 48 h (Fig. 4(b)). Fibrous blends exhibited a moderate release rate, accompanied by a rapid onset release $(65 \pm 1.7 \%$ at 10 $\min )$ and then a sustained phase $(70.0 \pm 1.2 \%$ for the following $48 \mathrm{hrs})$. Due to the highly water soluble nature of PVP, spun fibers dissolved simultaneously in PBS ensuring rapid active release. In contrast, PS is hydrophobic, and so composites of this and PVP provide a method to control active release; ideally suited for potential medicated fibrous device development (e.g. sustained wound dressing). For example, rapid active release is capable of killing bacteria at the wound site, and subsequent sustained release is ideal to inhibit bacterial growth [47]. In addition, numerous studies have shown cell growth (and therefore tissue repair) is enhanced using aligned fibers.

\subsection{Fiber ratio regulation}

A method to regulate the ratio of PS and PVP as blended fibers is demonstrated using a spinneret capable of hosting two or more formulated compartments. In this instance, the spinneret is shown to host four compartments, providing increased fiber production rate and improved blend integrity and control. While the compartment is adjustable, material selection adds to the possibilities of CMCCE. In the first instance a combinatorial selection (using two polymeric solutions i.e. PVP and PS) provides increased output and controlled fibrous composite gradient ratio blending. Fibrous blended mats comprising various PVP ratios (low to high as 1:1, 2:1, 3:1) are shown using gray-scale histogram scans (Figs. 5(a)-(c)). Fig. 5(a) shows composite fibers 
generated using a 1:1 ratio. PS fibers are observed as relative dark filaments, whilst PVP fibers appear closer to transparent. The gray-scale histogram scans were processed using a normalized background and image intensification. Here, lower gray values (yellow bar) correspond to darker regions (PS fibers) and higher values (red bar) correspond to lighter regions (PVP fibers) in histograms for micrographs. Increasing PVP compartments from 1 to 2 to 3 in the hosting spinneret, leads to an increase in the red histogram detection bar (gray-scale) as shown in Figs. 5(b) and (c). In addition, by comparing histograms for all groups, it is evident that PVP fiber quantity varies depending on the initial compartment ratio. This demonstrates an important and facile route to control fibrous mat production rate in general, and fiber composition in lieu.

\subsection{Diversity of material combination}

To elucidate further mat complexity and compositional fiber integration using CMCCE; a series of experiments were performed using a range of polymers with variable hydrophobic properties. Using the example shown in Figs. 6(a)-(g), samples comprising PCL, PS, PVP and TPU fibers, are prepared individually or integrated as a co-polymer blended system, Figs. 6(a)-(d) show optical micrographs of spun PCL, PS, PVP, and TPU mats, respectively. Fibers exhibit near uniform size distributions and appreciable alignment. FFT analysis (based on optical micrographs) appearing as insets in micrographs, show significant intensities indicating ordered fiber orientation. Fig. 6(e) shows aligned PCL, PVP fiber blends, Fig. 6(f) shows aligned PS-PVP blended fibers, and Fig. 6(g) shows aligned TPU and PVP fiber systems, which again indicates an excellent route to prepare multi-aligned fibers with varying surface and size properties.

\section{Conclusions}

In summary, we present a directional advance in engineering blended and multialigned fibers using multiple compartments and a modified spinneret making use of 
electrical and centrifugal forces. This method provides an opportunistic approach to prepare aligned fibrous matrices comprising two or more materials. Although a combinatorial system (for PS/PVP) was exemplified and quantified, the system is applicable to almost any polymeric material providing the viscoelastic properties are known. The present work demonstrates a promising low-cost and facile approach for large-scale production of stacked and blended fibers, with current explorations exhibiting implications in drug delivery, tissue engineering, food technology and smart functional material fabrication. The current proof of concept will now focus on the development and advancement of specific biomaterial applications.

\section{Acknowledgements}

This work was financially supported by the Key Technologies R\&D Program of Zhejiang Province(2015C02035) and the Fundamental Research Funds for the Central Universities (2017QNA5017).

\section{References}

[1] M.G. Mckee, T.E. Long, Phospholipid nonwoven electrospun membranes, Science, 311 (2006) 353-355.

[2] J.J. Li, L.T. Zhu, Z.H. Luo, Electrospun fibrous membrane with enhanced swithchable oil/water wettability for oily water separation, Chemical Engineering Journal, 287 (2015) 474-481.

[3] J. Xue, M. He, H. Liu, Y. Niu, A. Crawford, P.D. Coates, D. Chen, R. Shi, L. Zhang, Drug loaded homogeneous electrospun PCL/gelatin hybrid nanofiber structures for anti-infective tissue regeneration membranes, Biomaterials, 35 (2014) 9395-9405.

[4] J.G. Merrell, S.W. Mclaughlin, T. Lu, C.T. Laurencin, A.F. Chen, L.S. Nair, Curcumin Loaded Poly(ع-Caprolactone) Nanofibers: Diabetic Wound Dressing with Antioxidant and Anti-inflammatory Properties, Clinical and Experimental Pharmacology and Physiology, 36 (2009) 1149-1156.

[5] X. Wang, B. Liu, R. Liu, Q. Wang, X. Hou, D. Chen, R. Wang, G. Shen, Fiber-based flexible all-solid-state asymmetric supercapacitors for integrated photodetecting system, Angewandte Chemie International Edition, 53 (2014) 1849-1853.

[6] V. Thavasi, G. Singh, S. Ramakrishna, Electrospun nanofibers in energy and environmental applications, Energy \& Environmental Science, 1 (2008) 205-221.

[7] G. Zheng, Y. Yang, J.J. Cha, S.S. Hong, Y. Cui, Hollow Carbon Nanofiber-Encapsulated Sulfur 
Cathodes for High Specific Capacity Rechargeable Lithium Batteries, Nano Letters, 11 (2015) 4462-4467.

[8] K. Zhang, L.L. Zhang, X.S. Zhao, J. Wu, Graphene/polyaniline nanofiber composites as supercapacitor electrodes, Chemistry of Materials, 22 (2010) 1392-1401.

[9] H. Wu, L. Hu, M.W. Rowell, D. Kong, J.J. Cha, J.R. Mcdonough, J. Zhu, Y. Yang, M.D. Mcgehee, Y. Cui, Electrospun Metal Nanofiber Webs as High-Performance Transparent Electrode, Nano Letters, 10 (2010) 4242-4248.

[10] C. Zhang, C. Gao, M.W. Chang, Z. Ahmad, J.S. Li, Continuous micron-scaled rope engineering using a rotating multi-nozzle electrospinning emitter, Applied Physics Letters, 109 (2016) 173105-118897.

[11] Y. Wang, R. Gao, P.P. Wang, J. Jian, X.L. Jiang, C. Yan, X. Lin, L. Wu, G.Q. Chen, Q. Wu, The differential effects of aligned electrospun PHBHHx fibers on adipogenic and osteogenic potential of MSCs through the regulation of PPAR $\gamma$ signaling, Biomaterials, 33 (2012) 485-493.

[12] X. Zhang, V. Aravindan, P.S. Kumar, H. Liu, J. Sundaramurthy, S. Ramakrishna, S. Madhavi, Synthesis of $\mathrm{TiO} 2$ hollow nanofibers by co-axial electrospinning and its superior lithium storage capability in full-cell assembly with olivine phosphate, Nanoscale, 5 (2013) 5973-5980.

[13] D. Edmondson, A. Cooper, S. Jana, D. Wood, M. Zhang, Centrifugal electrospinning of highly aligned polymer nanofibers over a large area, Journal of Materials Chemistry, 22 (2012) 18646-18652.

[14] J.-C. Wang, H. Zheng, M.-W. Chang, Z. Ahmad, J.-S. Li, Preparation of active 3D film patches via aligned fiber electrohydrodynamic (EHD) printing, Scientific reports, 7 (2017) 43924.

[15] C. Niu, J. Meng, X. Wang, C. Han, M. Yan, K. Zhao, X. Xu, W. Ren, Y. Zhao, X. Lin, General synthesis of complex nanotubes by gradient electrospinning and controlled pyrolysis, Nature Communications, 6 (2015) 7402.

[16] G.T. Zhu, X.S. Li, X.M. Fu, J.Y. Wu, B.F. Yuan, Y.Q. Feng, Electrospinning-based synthesis of highly ordered mesoporous silica fiber for lab-in-syringe enrichment of plasma peptides, Chemical Communications, 48 (2012) 9980.

[17] G. Chen, Y. Xu, D.G. Yu, D.F. Zhang, N.P. Chatterton, K.N. White, Structure-tunable Janus fibers fabricated using spinnerets with varying port angles, Chemical Communications, 51 (2015) 4623.

[18] A.E. Erickson, D. Edmondson, F.C. Chang, D. Wood, A. Gong, S.L. Levengood, M. Zhang, High-throughput and high-yield fabrication of uniaxially-aligned chitosan-based nanofibers by centrifugal electrospinning, Carbohydrate Polymers, 134 (2015) 467-474.

[19] D. Brahatheeswaran, A. Mathew, R.G. Aswathy, Y. Nagaoka, K. Venugopal, Y. Yoshida, T. Maekawa, D. Sakthikumar, Hybrid fluorescent curcumin loaded zein electrospun nanofibrous scaffold for biomedical applications, Biomedical Materials, 7 (2012) 045001.

[20] X.Z. Sun, G.R. Williams, X.X. Hou, L.M. Zhu, Electrospun curcumin-loaded fibers with potential biomedical applications, Carbohydrate Polymers, 94 (2013) 147-153.

[21] A.J. Hassiba, M.E. El Zowalaty, G.K. Nasrallah, T.J. Webster, A.S. Luyt, A.M. Abdullah, A.A. Elzatahry, Review of recent research on biomedical applications of electrospun polymer nanofibers for improved wound healing, Nanomedicine, 11 (2016) 715-737.

[22] M.W. Rd, S.S. Murphree, J.E. Saffitz, Beta-adrenergic receptor distribution among muscle fiber types and resistance arterioles of white, red, and intermediate skeletal muscle, Circulation Research, 64 (1989) 1096-1105.

[23] A.R. Nielsen, R. Mounier, P. Plomgaard, O.H. Mortensen, M. Penkowa, T. Speerschneider, H. 
Pilegaard, B.K. Pedersen, Expression of interleukin - 15 in human skeletal muscle \&ndash; effect of exercise and muscle fibre type composition, Journal of Physiology, 584 (2007) 139618.

[24] A. Greiner, J.H. Wendorff, Electrospinning: a fascinating method for the preparation of ultrathin fibers, Angewandte Chemie International Edition, 46 (2007) 5670-5703.

[25] C. Liu, J. Sun, M. Shao, B. Yang, A comparison of centrifugally-spun and electrospun regenerated silk fibroin nanofiber structures and properties, Rsc Advances, 5 (2015) 98553-98558.

[26] L.D. Wright, R.T. Young, T. Andric, J.W. Freeman, Fabrication and mechanical characterization of 3D electrospun scaffolds for tissue engineering, Biomedical Materials, 5 (2010) 055006.

[27] A. Periyakaruppan, R.P. Gandhiraman, M. Meyyappan, J.E. Koehne, Label-Free Detection of Cardiac Troponin-I Using Carbon Nanofiber Based Nanoelectrode Arrays, Analytical Chemistry, 85 (2013) 3858-3863.

[28] Z. Yin, X. Chen, J.L. Chen, W.L. Shen, T.M. Hieu Nguyen, L. Gao, H.W. Ouyang, The regulation of tendon stem cell differentiation by the alignment of nanofibers, Biomaterials, 31 (2009) 2163-2175.

[29] T.K. Hiong Teh, G.J. Hong, S.L. Toh, Controlled Bioactive Molecules Delivery Strategies for Tendon and Ligament Tissue Engineering using Polymeric Nanofibers, Current Pharmaceutical Design, 21 (2015) 1991-2005.

[30] R.A. Neal, S.G. Mcclugage, M.C. Link, L.S. Sefcik, R.C. Ogle, E.A. Botchwey, Laminin nanofiber meshes that mimic morphological properties and bioactivity of basement membranes, Tissue Engineering Part C Methods, 15 (2009) 11-21.

[31] S.Y. Chew, R. Mi, A. Hoke, K.W. Leong, Aligned Protein-Polymer Composite Fibers Enhance Nerve Regeneration: A Potential Tissue-Engineering Platform, Advanced Functional Materials, 17 (2007) 1288-1296.

[32] B.W. Han, M.E. Mullins, J.M. Cregg, A. Hurtado, M. Oudega, M.T. Trombley, R.J. Gilbert, Creation of highly aligned electrospun poly-L-lactic acid fibers for nerve regeneration applications, Journal of Neural Engineering, 6 (2009) 643-653.

[33] M.M.L. Arras, C. Grasl, H. Bergmeister, H. Schima, Electrospinning of aligned fibers with adjustable orientation using auxiliary electrodes, Science and Technology of Advanced Materials, 13 (2012) 35008-35015.

[34] M. Inagaki, Y. Yang, F. Kang, Carbon nanofibers prepared via electrospinning, Advanced Materials, 24 (2012) 2547-2566.

[35] N.M. Thoppey, J.R. Bochinski, L.I. Clarke, R.E. Gorga, Edge electrospinning for high throughput production of quality nanofibers, Nanotechnology, 22 (2011) 486-498.

[36] L. Wang, M.W. Chang, Z. Ahmad, H. Zheng, J.S. Li, Mass and controlled fabrication of aligned PVP fibers for matrix type antibiotic drug delivery systems, Chemical Engineering Journal, 307 (2016) 661-669.

[37] K.L. Menzies, L. Jones, The impact of contact angle on the biocompatibility of biomaterials, Optometry \& Vision Science Official Publication of the American Academy of Optometry, 87 (2010) 387.

[38] Z.G. Tang, R.A. Black, J.M. Curran, J.A. Hunt, N.P. Rhodes, D.F. Williams, Surface properties and biocompatibility of solvent-cast poly[-caprolactone] films, Biomaterials, 25 (2004) 4741.

[39] T. Tsukagoshi, Y. Kondo, N. Yoshino, Preparation of thin polymer films with controlled drug release, Colloids \& Surfaces B Biointerfaces, 57 (2007) 219-225.

[40] X. Wang, T. Lin, Electric field analysis of spinneret design for needleless electrospinning of 
nanofibers, J. Mater. Res., 27 (2012) 3013-3019.

[41] X. Wang, X. Wang, T. Lin, 3D electric field analysis of needleless electrospinning from a ring coil, J. Ind. Text., 44 (2013) 463-476.

[42] H. Tang, T. Cao, X. Liang, A. Wang, S.O. Salley, M.A.J. Nd, K.Y. Ng, Influence of silicone surface roughness and hydrophobicity on adhesion and colonization of Staphylococcus epidermidis, Journal of Biomedical Materials Research Part A, 88A (2009) 454-463.

[43] M. Essalhi, M. Khayet, Self-sustained webs of polyvinylidene fluoride electrospun nano-fibers: Effects of polymer concentration and desalination by direct contact membrane distillation, Journal of Membrane Science, 454 (2014) 133-143.

[44] N. Sarlak, M.A.F. Nejad, S. Shakhesi, K. Shabani, Effects of electrospinning parameters on titanium dioxide nanofibers diameter and morphology: An investigation by Box-Wilson central composite design (CCD), Chemical Engineering Journal, 210 (2012) 410-416.

[45] X. Liang, D. Huang, New Insights into the Effects of Physical Aging on Glass Transition of Polystyrene by FTIR, Journal of Macromolecular Science Part B Physics, 51 (2012) 348-357.

[46] Y. Zhou, P. Qi, Z. Zhao, Q. Liu, Z. Li, Fabrication and characterization of fibrous HAP/PVP/PEO composites prepared by sol-electrospinning, Rsc Advances, 4 (2014) 16731-16738.

[47] J.B. Deris, M. Kim, Z. Zhang, H. Okano, R. Hermsen, A. Groisman, T. Hwa, The Innate Growth Bistability and Fitness Landscapes of Antibiotic-Resistant Bacteria, Science, 342 (2013) 1237435. 


\section{Figures captions}

Fig. 1. Schematic of the centrifugal electrospinning system and fabricated fibers. (a) Schematic diagram of the complete CMCCE system. (b) Schematic diagram of the hosting spinneret detached from two compartments. (c) Spinneret hosting four compartments in a 3:1 ratio (d) Computational detail (EFM) of two individual jets (each from their respective solution compartments) emerging under an applied electric field. (e) Illustration of a composite material resulting from CMCCE and (f) fluorescent micrograph of a blended system comprising PVP (rhodamine-B) and PS (acriflavine) (CMCCE conditions deployed $400 \mathrm{rpm}, 18 \mathrm{kV}$ ).

Fig. 2. Optical, electron and fluorescent micrographs of aligned fibers obtained using different materials. (a)(d)(g) PS, (b)(e)(h) PVP and (c)(f)(i) both PS and PVP solutions, respectively. Corresponding fiber diameter distribution and water contact angles are shown in $(\mathrm{j})(\mathrm{k})(\mathrm{l})$, respectively.

Fig. 3. The analysis of working parameters. (a) Relationship between viscosity and polymer concentration, with fiber quality zones mapped on plot surface. (b) Depiction of viable working voltage and rotation speed for PS and PVP polymer compartments. Mean PS and PVP fiber diameter distribution modulated using various working parameters: (c) solution concentration (d) compartment orifice size (e) spinneret rotation speed and (f) applied voltage.

Fig. 4. Study on the biomedical properties of the fabricated fibers. (a) FTIR spectra of (I) TE-HCL ( II) PS (III) PVP (IV) PS \& TE-HCL ( V) PVP \& TE-HCL (VI) PS \& PVP \& TE-HCL. (b) cumulative TE-HCL release from PS, PVP, PS \& PVP fibrous samples over time.

Fig. 5. Ratio control. Optical micrographs and gray scale analysis of fibers with different ratio of PS and PVP by using different number of working solution tubes: (a) 1 PS tube: 1 PVP tube. (b) 1 PS tube: 2 PVP tubes. (c) 1 PS tube: 3 PVP tubes.

Fig. 6. Material type control. Optical micrographs and FFT analysis of fibers fabricated using CMCCE (400 rpm and $18 \mathrm{kV}$ ) from material (a) PCL (b) PS (c) PVP (d) TPU. Blended fibers produced from combined materials (e) PCL and PVP (f) PS and PVP (g) TPU and PVP. 


\section{Figures}

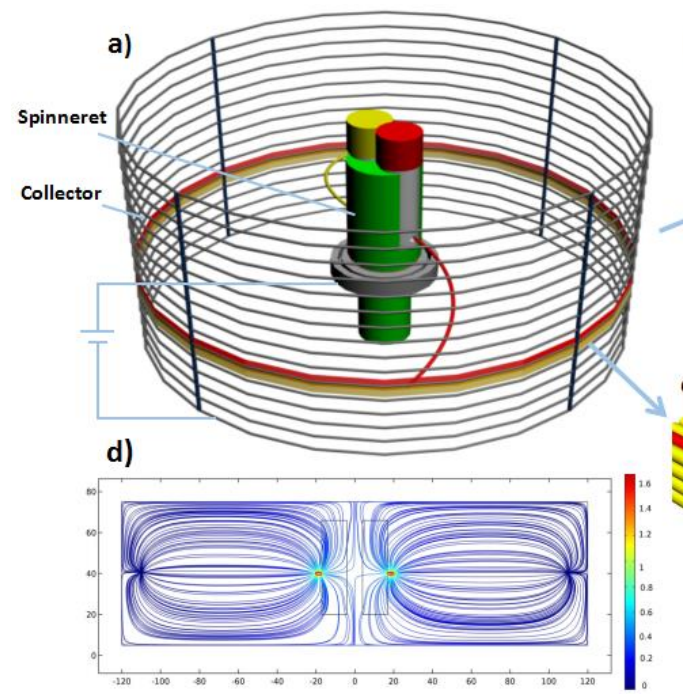

b)

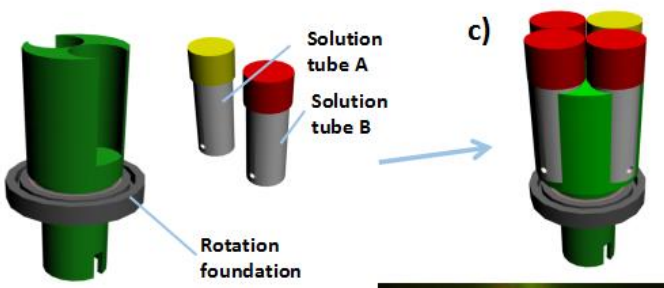

e)

Blended fibers
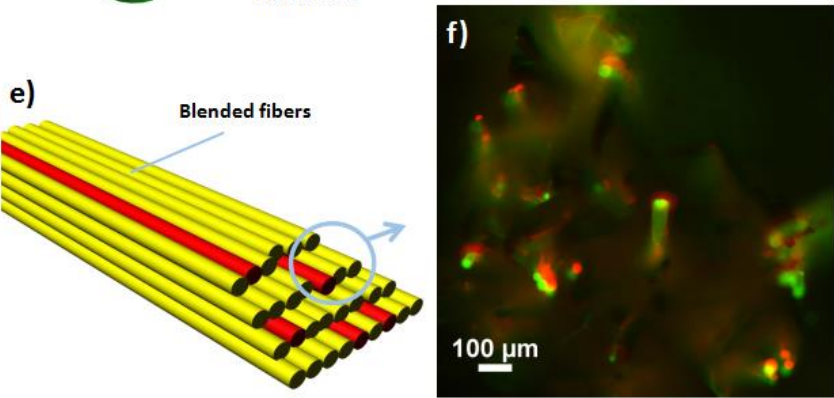

Fig. 1. 

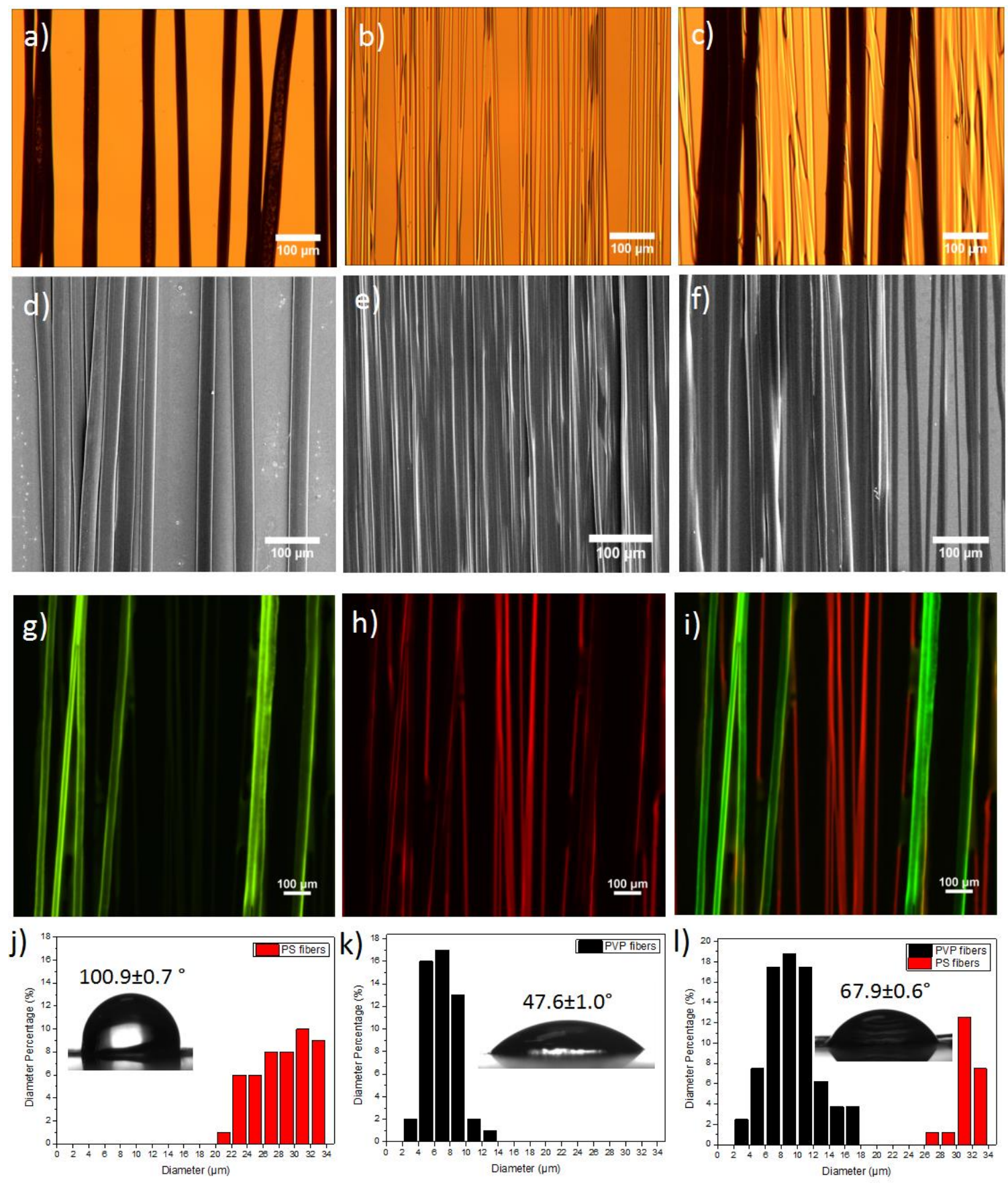

Fig. 2. 
a)

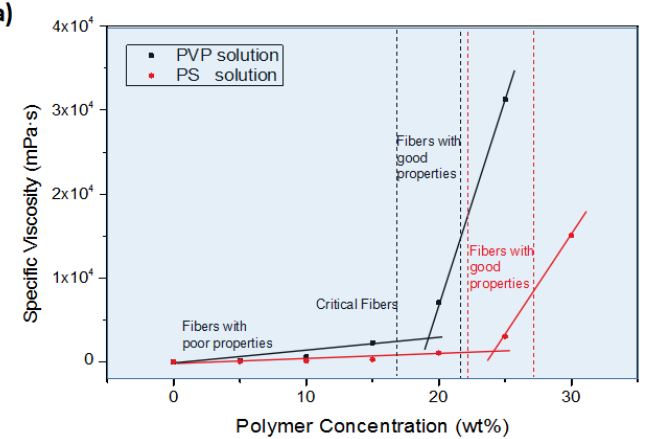

b)
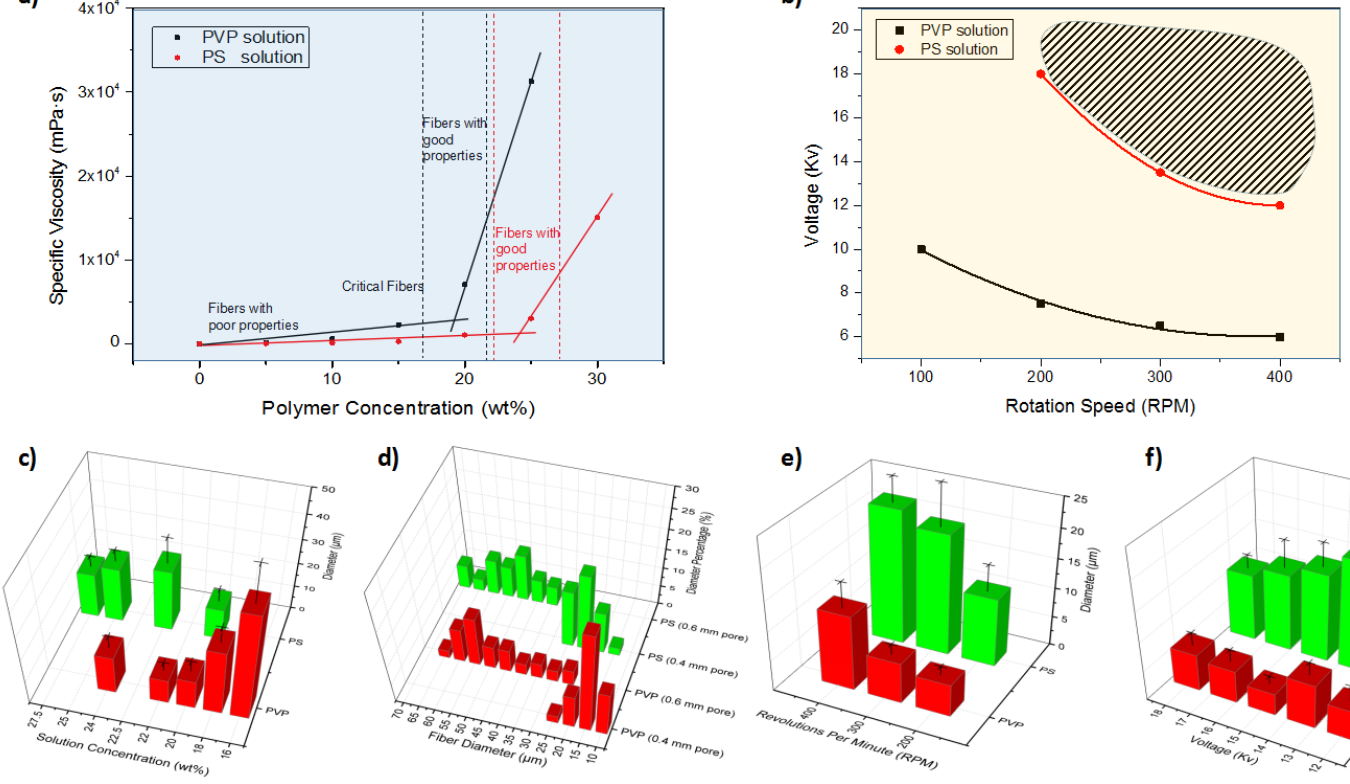

d)

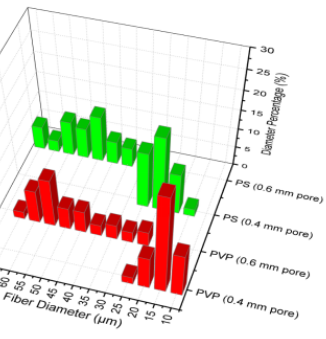

e)

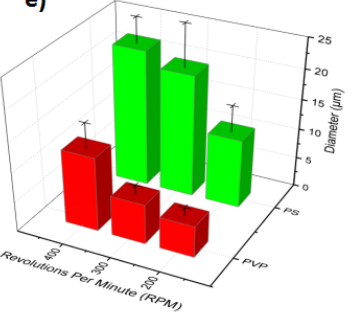

f)

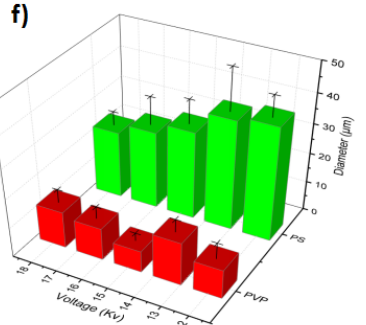

Fig. 3. 
a)

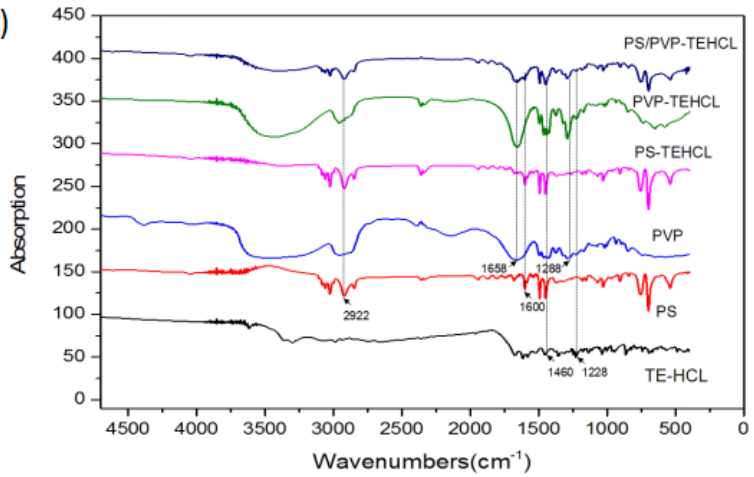

b)

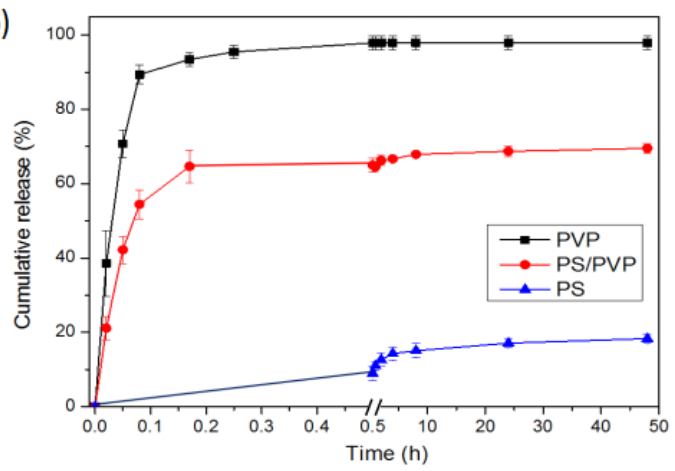

Fig. 4.
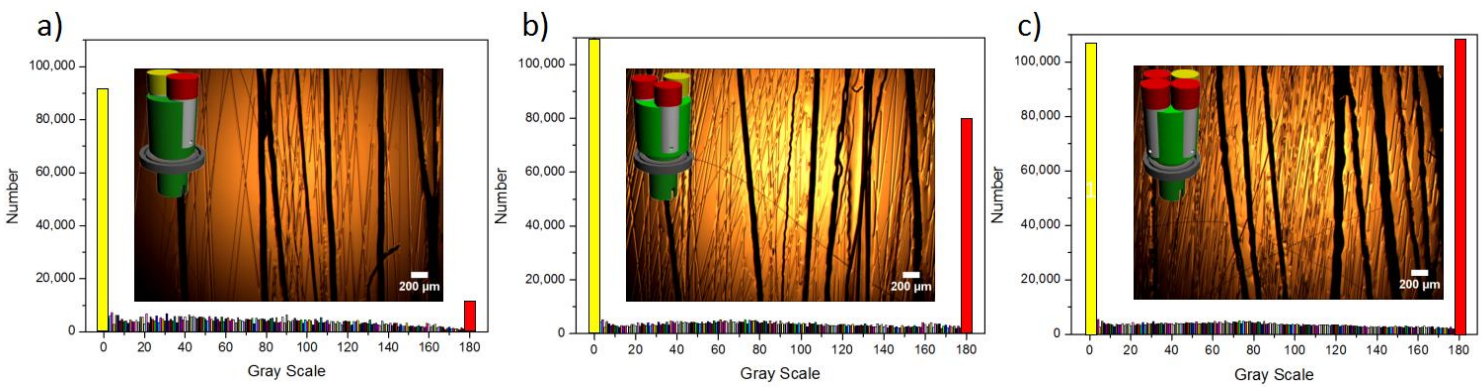

Fig. 5. 

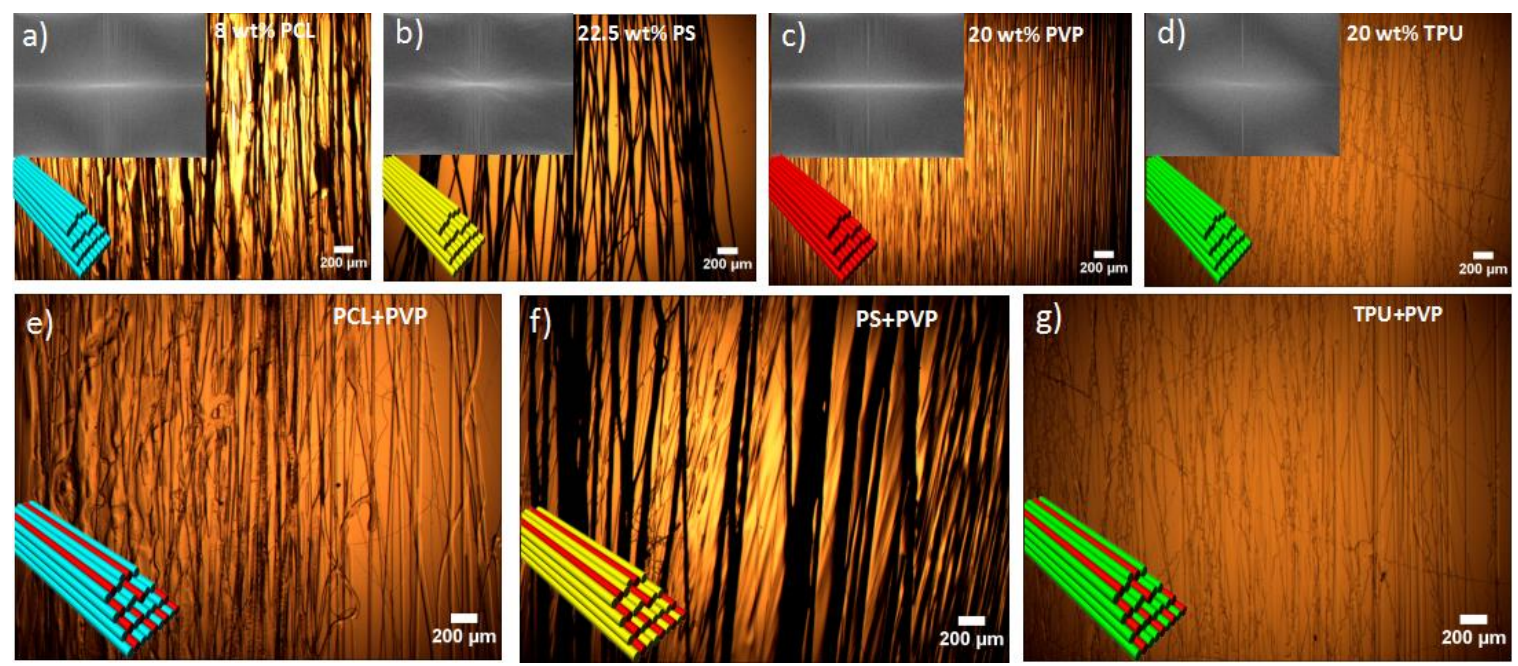

g)

TPU+PVP

Fig. 6. 